\title{
Enhancement of the microwave magneto-Kerr effect in semiconductors using dielectric matching techniques
}

\author{
W. F. Perger \\ Department of Physics, Colorado State University, Fort Collins, Colorado 80523
}

R. J. Vernon and C. M. Lee

Department of Electrical Engineering, University of Wisconsin-Madison, Madison, Wisconsin 53706

(Received 22 March 1985; accepted for publication 14 May 1985)

\begin{abstract}
Enhancement of the microwave magneto-Kerr effect in semiconductors is discussed for the semiconductor being preceded by a semi-infinite dielectric and by a quarter-wave dielectric slab for both plane-wave and guided-wave cases. Expressions are developed that relate the amount of the enhancement to the relative permittivity of the dielectric. Experimental results are compared to the theoretically obtained expressions for the quarter-wave case and the experimentally obtained values compare favorably with the theory.
\end{abstract}

\section{INTRODUCTION}

When a linearly polarized electromagnetic wave is normally incident on a semiconducting slab that is immersed in a static magnetic field, the transmitted and reflected waves will, in general, be elliptically polarized with the major axis of the ellipse rotated relative to the incident plane of polarization. This is termed the Faraday effect in the transmitted wave and the magneto-Kerr effect in the reflected wave. The magneto-Kerr effect has received much attention in investigating semiconductor transport parameters. ${ }^{l}$ It was discussed by Lax and Zwerdling ${ }^{2}$ in 1960 and observed by Brodwin et $a l .{ }^{3}$ in 196\%. It has been analyzed and measured by Brodwin and Vernon' in terms of $R$ and $\delta$, the amplitude and phase, respectively, of the ratio of the two orthogonally polarized components of the refiected wave. The method of measuring the magneto-Kerr effect used in this study was discussed by Vernon and Dorschner. ${ }^{4}$

Although the magneto-Kerr effect is often more useful for semiconductors with high conductivity than the Faraday effect because the latter depends on the transmission of radiation, the measurement of the magneto-Xerr effect may also become difficult for semiconductors in which the ratio of Hall mobility to static conductivity is small.

It is shown here both theoretically and experimentally that the magneto-Kerr effect may be enhanced by placing dielectric materials in front of the semiconductor. Analysis is given for the case of normal incidence of a plane wave and for a $T E_{11}$ mode in a circular waveguide. The exact expressions for $R$ as well as approximate expressions for the case of high-loss semiconductors, low magnetic fields, and small $\omega \tau$ are given ( $\omega$ is the angular frequency of the incident electromagnetic field and $\tau$ is the semiconductor scattering time).

\section{月1. THEORY OF THE MAGNETO-KERR EFFECT}

In this study the semiconductor is treated as having a complex conductivity associated with the free carriers as well as having dielectric properties. When a static magnetic field is applied along the [100] or [111] crystallographic direction of $n$-type germanium or $n$-type silicon, the conductivity tensor $\sigma$ for high-frequency electric fields may be written in the form for a magnetic field in the $z$ direction as ${ }^{5}$

$$
\boldsymbol{\sigma}=\left(\begin{array}{ccc}
\sigma_{11} & \sigma_{12} & 0 \\
-\sigma_{12} & \sigma_{11} & 0 \\
0 & 0 & \sigma_{33}
\end{array}\right) .
$$

This form of tensor is valid for any magnetic field orientation with respect to crystallographic axis if the constant energy surfaces for the carrier are spherical.

If we now consider the case of a plane wave propagating in a semiconductor with permittivity $\epsilon_{s}$, permeability $\mu_{0}$, and conductivity tensor $\sigma$, then the normal modes of propagation are ${ }^{6}$

$$
\mathbf{E}_{ \pm}=\left(\begin{array}{c}
\mathbf{E}_{0} \\
\mp i \mathbf{E}_{0}
\end{array}\right) \exp \left(-i k_{ \pm} z\right)
$$

where

$$
k_{ \pm}^{2}=\omega^{2} \epsilon_{s} \mu_{0}-i \omega \mu_{0} \sigma_{ \pm},
$$

and

$$
\sigma_{ \pm}=\sigma_{11} \mp i \sigma_{12}, \quad(\text { where } i=\sqrt{-1}) .
$$

Note that $\mathbb{E}_{+}$and $E_{-}$are right-hand and left-hand circularly polarized waves, respectively, for propagation in the positive $z$ direction.

A linearly polarized plane wave may be considered to be a superposition of two counter-rotating circularly polarized waves. Since these component waves are the normal modes in the semiconductor, the reflection of a linearly polarized plane wave normally incident upon the sample may be easily analyzed by considering them separately.

Assume that the incident wave is polarized in the $x$ direction and that a static magnetic field is applied along the $z$ direction, the direction of propagation. Then the electric field vectors for each component wave are

$$
\mathbf{E}_{i \pm}=\frac{1}{2} E_{0}\left(\hat{a}_{x} \mp i \hat{a}_{y}\right) \exp (-i k z),
$$

where $\mathbf{E}_{i+}$ and $\mathbf{E}_{i-}$ are the vector phasors for the righthand and the left-hand circularly polarized waves respectively. Since the conductivities seen by the two normal modes are $\sigma_{ \pm}$as given by (3), each component wave sees a different reflection coefficient given by

$$
\rho_{ \pm}=\frac{E_{r \pm}}{E_{i_{ \pm}}}=\frac{\eta_{s_{ \pm}}-\eta_{0}}{\eta_{s \pm}+\eta_{0}}
$$


where $\eta_{s_{ \pm}}$are the two complex intrinsic wave impedances of the semiconductor corresponding to $\sigma_{ \pm}$, and $E_{r_{ \pm}}$are the reflected electric field components corresponding to $E_{i_{ \pm}}$. The reflected wave components are

$$
\mathbf{E}_{r \pm}=\frac{1}{2} E_{0}\left(\hat{a}_{x} \mp i \hat{a}_{y}\right) p_{ \pm} \exp (i k z) \text {. }
$$

The reflected right- and left-hand circularly polarized waves may be reexpressed in terms of the orthogonal Cartesian components:

$$
\begin{aligned}
& E_{r x}=\frac{1}{2} E_{0}\left(\rho_{+}+\rho_{-}\right) \exp (i k z), \\
& E_{r y}=\frac{1}{2} E_{0} i\left(\rho_{+}-\rho_{-}\right) \exp (i k z) .
\end{aligned}
$$

The total reflected wave will thus have two orthogonally polarized wave components resulting in an elliptically polarized wave in general.

Let $R$ be the magnitude of the ratio of the two orthogonal components of the reflected wave and $\delta$ the phase difference between them as was done by Brodwin and Vernon. ${ }^{1}$ Then:

$$
\begin{aligned}
R e^{i \delta} & =E_{r y} / E_{r x} \\
& \left.=-i \llbracket\left(\rho_{+}-\rho_{-}\right) /\left(\rho_{+}+\rho_{-}\right)\right] .
\end{aligned}
$$

Hence

$$
R=\left|\left(\rho_{+}-\rho_{-}\right) /\left(\rho_{+}+\rho_{-}\right)\right|
$$

and

$$
\delta=-\pi / 2+\arg \left[\left(\rho_{+}-\rho_{-}\right) /\left(\rho_{+}+\rho_{-}\right)\right] .
$$

Brodwin and Vernon ${ }^{1}$ have given an approximate expression for $R$ for the case of

$$
\omega \epsilon_{s} / \sigma_{s}<1, \quad \mu B<1 \text {, and } \omega \tau<1
$$

as

$$
R=\mu B\left(\omega \epsilon_{0} / \sigma_{s}\right)^{1 / 2},
$$

where $\mu$ is the Hall mobility, $\epsilon_{s}$ the static permittivity, and $\sigma_{s}$ the dc conductivity of the semiconductor. $B$ is the static magnetic flux density. It is seen from Eq. (9) that $R$ becomes very small when $\mu$ becomes small and $\sigma_{s}$ becomes large thereby making the measurement of $R$ difficult. Therefore, it is desirable to increase $R$ in the above cases to be able to measure the semiconductor parameters with accuracy while reducing concern for the minimum detectable $R$.

\section{HI. PLANE WAVE PROPAGATION}

\section{A. Enhancement of $R$}

As previously discussed, when the sample conductivity is large and the Hall mobility is small, a measurement of $R$ is difficult. It is possible, however, to couple more energy into the semiconductor and increase the free carrier interaction with the incoming electromagnetic wave. This increase in the coupling of energy may be achieved through an impedance transformation by a dielectric material. The magnetoKerr effect is enhanced as the reflected wave component with the polarization orthogonal to that of the incident wave increases while the reflected wave component with the polarization parallel remains aimost unchanged.

\section{B. Semi-infinite dielectric space}

Now consider the case of semi-infinite dielectric space with permittivity $\epsilon_{2}$ preceding the semiconductor. For this case the formulation of $R$ and $\delta$ of the magneto-Kerr effect is identical to that of the free-space case discussed earlier except that $\epsilon_{0}$ must be replaced by $\epsilon_{2}$ in Eq. (9). Note, however, that the enhancement of $R$ increases only as the square root of the relative permittivity.

\section{General development of dielectric enhancement}

Consider the case of a plane polarized wave normally incident upon a semiconductor surface with a dielectric medium in front as shown in Fig. 1. Assume that the surfaces of the dielectric and semiconductor are planes perpendicular to the $z$ direction and that the incident electric field is polarized in the $x$ direction and the static magnetic field is applied in the $z$ direction.

Each of the counter-rotating components of the incident plane wave sees a different reflection coefficient at $z=d$ and $z=0$. The intrinsic wave impedances of the semiconductor for these two circularly polarized waves are

$$
Z_{ \pm}=\left[i \omega \mu_{0} /\left(\sigma_{ \pm}+i \omega \epsilon_{s}\right)\right]^{1 / 2}
$$

where $\sigma_{ \pm}$of Eq. (3) can be written ${ }^{6}$ as

$$
\sigma_{ \pm}=\sigma_{s} /[1 \pm i(\omega \tau \pm \mu B)] \text {. }
$$

Assuming that the dielectric medium is lossless and making use of simple transmission line theory for the transformation of impedances, one can obtain the impedance seen at $z=d$ :

$$
Z_{i \pm}=\left(\frac{\mu_{0}}{\epsilon_{2}}\right)^{1 / 2}\left(\frac{\cos \beta_{2} d+i \zeta \sin \beta_{2} d}{\zeta \cos \beta_{2} d+i \sin \beta_{2} d}\right),
$$

where $\beta_{2}$ is the phase constant in the dielectric medium and

$$
\xi=\left[\left(\epsilon_{s}-i \frac{\sigma_{ \pm}}{\omega}\right) \epsilon_{2}\right]^{1 / 2}
$$

The refiection coefficients at $z=d$ are then given by

$$
\rho_{ \pm}=\left(Z_{i \pm}-\eta_{0}\right) /\left(Z_{i_{ \pm}}+\eta_{0}\right)
$$

where $\eta_{0}=\left(\mu_{0} / \epsilon_{0}\right)^{1 / 2}$ is the intrinsic impedance of free space. Then the amplitude ratio $R$ of the two orthogonal components of the reflected wave at $z=d$ may be obtained from Eq. (7)

$$
R=\frac{\left(1-1 / \xi_{i_{+}}\right)\left(1+1 / \xi_{i_{-}}\right)-\left(1+1 / \xi_{i_{+}}\right)\left(1-1 / \xi_{i_{-}}\right)}{\left(1-1 / \xi_{i_{+}}\right)\left(1+1 / \xi_{i_{-}}\right)+\left(1+1 / \xi_{i_{+}}\right)\left(1-1 / \xi_{i_{-}}\right)},
$$

where

$$
\xi_{i \pm}=Z_{i \pm} / \eta_{0}
$$



FIG. 1. Dielectric material of thickness $d$ preceding the semiconductor. 


\section{Quarter-wave dielectric}

Now consider the case shown in Fig. 1 again and let $d=\lambda_{2} / 4$, where $\lambda_{2}$ is the wavelength in the dielectric medium. Then Eq. (11) becomes

$$
Z_{i \pm}=\frac{\eta_{0}}{\epsilon_{2 r}}\left(\epsilon_{s r}-i \frac{\sigma_{ \pm}}{\omega \epsilon_{0}}\right)^{1 / 2},
$$

where $\epsilon_{s r}$ and $\epsilon_{2 r}$ are the relative permittivities for the dielectric and semiconducting regions, respectively. From Eq. (14),

$$
\xi_{i \pm}=\frac{1}{\epsilon_{2 r}}\left(\epsilon_{\mathrm{sr}}-i \frac{\sigma_{ \pm}}{\omega \epsilon_{0}}\right)^{1 / 2}
$$

substituting Eq. (16) for $\xi_{i \pm}$, Eq. (13) can be written as

$$
R=\left|\frac{\left[\epsilon_{2 r} /\left(i \omega \epsilon_{0}\right)\right]\left[\left(\sigma_{+}+i \omega \epsilon_{s}\right)^{1 / 2}-\left(\sigma_{-}+i \omega \epsilon_{s}\right)^{1 / 2}\right]}{\frac{\left(\sigma_{+} \sigma_{-}\right)^{1 / 2}}{i \omega \epsilon_{0}}\left[\left(1+i \frac{\omega \epsilon_{s}}{\sigma_{+}}\right)\left(1+i \frac{\omega \epsilon_{s}}{\sigma_{-}}\right)\right]^{1 / 2}-\epsilon_{s r}^{2}}\right|
$$

\section{GUIDED WAVE PROPAGATION}

\section{A. General description}

The magneto-Kerr effect is difficult to measure in plane waves at microwave frequencies, and since the two orthogonally polarized waves of the dominant mode can propagate equally well in a circular waveguide, measurements are made for the $T E_{11}$ mode in a circular waveguide using a dual mode transducer. ${ }^{4}$

Consider the case of the dominant $\mathrm{TE}_{11}$ mode excited in a circular waveguide filled with an isotropic semiconductor with a lossiess dielectric material in front as shown in Fig. 2. Assume that a small static magnetic field is applied longitudinally in the $z$ direction. Only the dominant modes need be considered here since all the higher modes which are generated at the dielectric-semiconductor interface have amplitudes second order or higher in magnetic field. ${ }^{7}$ Champlin $^{8}$ gives the propagation constants for the two counter-rotating circularly polarized $\mathrm{TE}_{11}$ waves in the semiconductor by employing perturbation methods for low magnetic fields $(\mu B<1)$ as

$$
K_{g \pm}^{2}=\omega^{2} \mu_{0} \epsilon_{s}-i \omega \mu_{0}\left(\sigma_{11 \mp} i \kappa \sigma_{12}\right)-K_{r}^{2},
$$

where $\kappa=0.838$, and $K_{c}$ is the cutoff wavenumber for the dominant mode of the guide which is $1.841 / a, a$ being the radius of the waveguide.

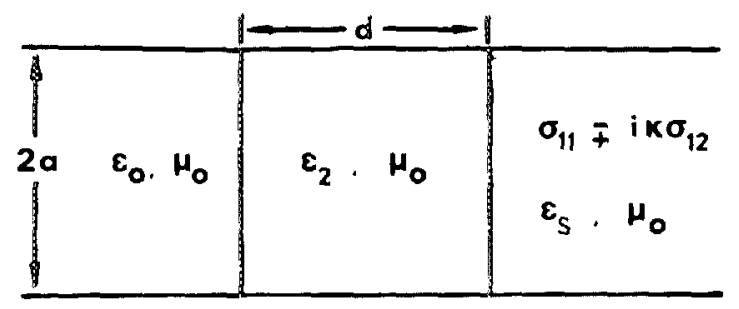

FIG. 2. A circular waveguide filled with a semiconductor and a dielectric disk of thickness $d$.

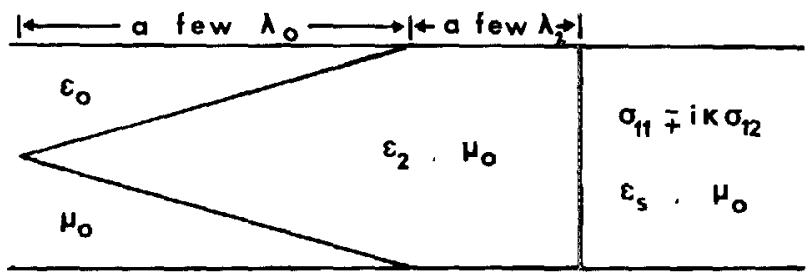

FIG. 3. Tapered dielectric rod preceding the semiconductor inside a circular waveguide.

\section{B. Slowly tapered dielectric rod}

First consider the hypothetical case of a semi-infinitely long dielectric rod which is placed in a circular waveguide preceding the semiconductor. This case is the guided wave analog of the plane-wave case which was discussed in Sec. III B.

Since it is difficult to arrange a semi-infinitely long dielectric rod inside a circular waveguide in a laboratory, a dielectric rod a few wavelengths long with one end slowly tapered would be used as an approximation for the semiinfinite rod. The case being described is shown in Fig. 3. The tapered section is assumed to be a few free-space wavelengths long and uniform so that there is negligible reflection of the incident wave before reaching the dielectric-semiconductor interface.

The complex wave impedance of the $T E_{11}$ mode in the semiconductor inside the circular waveguide is

$$
Z_{g \pm}=\omega \mu_{0} / K_{g \pm},
$$

where $K_{g \pm}$ is given by Eq. (18) and the wave impedance for the $\mathrm{TE}_{11}$ mode in the circular dielectric rod is

$$
Z_{g 2}=\omega \mu_{0} /\left(K_{0}^{2} \epsilon_{2 r}-K_{c}^{2}\right)^{1 / 2},
$$

where $K_{0}=\omega\left(\mu_{0} \epsilon_{0}\right)^{1 / 2}$ is the free-space wavenumber. The reflection coefficients for the two counter-rotating circularly polarized waves are

$$
\rho_{g \pm}=\left(Z_{g \pm}-Z_{g^{2}}\right) /\left(Z_{g \pm}+Z_{g^{2}}\right) \text {. }
$$

$R_{\mathrm{g}}$ and $\delta_{\mathrm{g}}$ are given by Eqs. (7) and (8). The subscript $g$ denotes the guided wave parameters:

$$
\begin{aligned}
& \left.R_{g}=\| \rho_{g+}-\rho_{g-}\right) /\left(\rho_{g+}+\rho_{g-}\right) \|, \\
& \delta_{g}=-\frac{\pi}{2}+\arg \left(\frac{\rho_{g+}-\rho_{g-}}{\rho_{g+}+\rho_{g-}}\right) .
\end{aligned}
$$

\section{Dielectric slabs of finite thlickness}

As was done in the plane-wave case, the transmission line analogy can be used to find the impedance seen at $z=d$ by the counter-rotating circularly polarized wave components of the incident wave. This input impedance, denoted by $Z_{i g \pm}$, seen at $z=d$ may be written as

$$
Z_{i g \pm}=\left(\frac{Z_{g \pm} \cos \left(K_{g 2} d\right)+i Z_{g^{2}} \sin \left(K_{g^{2}} d\right)}{Z_{g^{2}} \cos \left(K_{g^{2}} d\right)+i Z_{g \pm} \sin \left(K_{g^{2}} d\right)}\right) Z_{g^{2}},
$$

where $K_{g 2}=\left(K_{0}^{2} \epsilon_{2 r}-K_{c}^{2}\right)^{1 / 2}$ is the phase constant in the dielectric and $d$ is the thickness of the dielectric slab. Then the two reflection coefficients corresponding to the normal modes are 


$$
\begin{aligned}
\rho_{g_{ \pm}} & =\left(Z_{i g_{ \pm}}-Z_{80}\right) /\left(Z_{i g \pm}+Z_{80}\right), \\
& =\left(1-1 / \xi_{i g \pm}\right) /\left(1+1 / \xi_{i g \pm}\right),
\end{aligned}
$$

where $Z_{g 0}$ is the wave impedance of the empty waveguide and is given by

$$
Z_{80}=\omega \mu_{0} /\left(K_{0}^{2}-K_{c}^{2}\right)^{1 / 2}
$$

and

$$
\xi_{i g \pm}=Z_{i g \pm} / Z_{80} .
$$

$R_{g}$ is given by substituting Eqs. (25)-(27) into (22).

\section{Quarter-wave dielectric}

For the special case of the dielectric slab being onequarter wavelength thick, $d=\lambda_{82} / 4$ in Eq. (24) where

$$
\begin{aligned}
\lambda_{g 2} & =2 \pi / K_{g 2}, \\
& =2 \pi /\left(K_{0}^{2} \epsilon_{2 r}-K_{c}^{2}\right)^{1 / 2} .
\end{aligned}
$$

For this case Eq. (24) becomes

$$
Z_{i \mathrm{~g} \pm}=\left(Z_{\mathrm{g}^{2}}\right)^{2} / Z_{\mathrm{g} \pm} \text {, }
$$

which, in turn, can be rewritten using Eqs. (19), (20), and (18) as

$$
Z_{i g \pm}=\frac{\omega \mu_{0}\left[\omega^{2} \mu_{0} \epsilon_{s}-i \omega \mu_{0}\left(\sigma_{11} \mp i \kappa \sigma_{12}\right)-K_{c}^{2}\right]^{1 / 2}}{K_{0}^{2} \epsilon_{2 r}-K_{c}^{2}} .
$$

\section{APPAOXIMATIONS FOR THE HIGH-LOSS PLANE- WAVE CASE}

\section{A. Descriptions of approximations}

For heavily doped semiconductors the static conductivity may be large. In addition, if the applied magnetic field is small and $\omega$ is in the millimeter microwave band or lower, then the following inequalities exist:

$$
\frac{\sigma}{\omega \epsilon_{s}}>1, \quad \mu B<1, \quad \omega \tau<1 .
$$

These were the approximations used by Brodwin and Vernon ${ }^{1}$ to obtain Eq. (9).

\section{B. Semí-infinite dielectric enhancement}

As was previously mentioned, the expression for $R$ in Eq. (9) is modified for the case of the semi-infinite dielectric by the use of $\epsilon_{2}=\epsilon_{2 r} \epsilon_{0}$ instead of $\epsilon_{0}$. Thus

$$
R \simeq \mu B\left(\omega \epsilon_{0} / \sigma_{s}\right)^{1 / 2} \epsilon_{2 r}^{1 / 2},
$$

where $\epsilon_{2 r}$ is the relative permittivity of the dielectric. It is clear from Eq. (30) that $R$ is enhanced by a factor of $\epsilon_{2 r}^{1 / 2}$.

\section{Quarter-wave case}

Using the approximations in Eq. (29), (10) becomes

$$
\begin{aligned}
\sigma_{ \pm} & =\sigma_{s} /[1+i(\omega \tau \pm \mu B)], \\
& \simeq \sigma_{s} /(1 \pm i \mu B), \\
& \simeq \sigma_{s}(1 \mp i \mu B) .
\end{aligned}
$$

Using Eq. (31) in (17) yields

$$
R \simeq \mu B\left(\omega \epsilon_{0} / \sigma_{s}\right)^{1 / 2} \epsilon_{2 r},
$$

when terms involving second or higher orders of $\mu B, \omega \epsilon_{0} / \sigma_{s}$, and $\omega \epsilon_{s} / \sigma_{s}$ are neglected. Notice that for this approximate expression (32), there exists a linear relationship between $\boldsymbol{R}$ and $\epsilon_{2 r}$. This approximation breaks down when the value of $\epsilon_{2 r}$ approaches $\left(\sigma_{s} / 2 \omega \epsilon_{0}\right)^{1 / 2}$. The functional relationship between $R$ and $\epsilon_{2 r}$ for the quarter-wave case becomes nonlinear when $R$ approaches its maximum. It can be shown that the maximum magneto-Kerr effect (the greatest $R$ ) is given by

$$
\epsilon_{2 r}=\left[\epsilon_{s r}^{2}+\left(\sigma_{s} / \omega \epsilon_{0}\right)^{2}\right]^{1 / 4}
$$

\section{V.. APPROXIMATIONS FOR THE HIGH-LOSS, GUIDED WAVE CASE}

\section{A. Tapered dielectric rod}

For the high-loss case being considered here, the two conductivity tensor elements $\sigma_{11}$ and $\sigma_{12}$ may be approximated by neglecting terms involving $\omega \tau$ and $(\mu B)^{2}$ as follows:

$$
\sigma_{11} \simeq \sigma_{s}, \quad \sigma_{12} \simeq \sigma_{s} \mu B .
$$

Hence, the propagation constants for the counter-rotating wave components of a $T E_{11}$ mode in a circular waveguide may be written from Eq. (18):

$$
\begin{aligned}
K_{8 \pm} & \simeq\left[K_{0}^{2} \epsilon_{s r}-K_{c}^{2}-i \omega \mu_{0} \sigma_{s}(1 \mp i \kappa \mu B)\right]^{1 / 2}, \\
& \simeq\left(-i \omega \mu_{0} \sigma_{s}\right)^{1 / 2}(1 \mp i l \kappa \mu B) .
\end{aligned}
$$

Thus, for the case of the tapered dielectric rod shown in Fig. 3, Eq. (21) becomes

$$
\rho_{\mathrm{g} \pm} \simeq-\left[1-2 Z_{\mathrm{g} \pm} / Z_{\mathrm{g} 2}\right] \text {. }
$$

Then $R_{g}$ is approximately given by

$$
R_{g} \simeq\left(\epsilon_{2 r}-K_{c}^{2} / K_{0}^{2}\right)^{1 / 2} \kappa \mu B\left(\omega \epsilon_{0} / \sigma_{s}\right)^{1 / 2} .
$$

If the enhancement factor $E_{\mathrm{g}}$ is defined as the ratio of the enhanced $R_{g}$ to the $R_{g}$ without dielectric enhancement, then $E_{8}$ for the tapered dielectric rod is

$$
E_{g} \simeq\left(\frac{\epsilon_{2 r}-K_{c}^{2} / K_{0}^{2}}{1-K_{c}^{2} / K_{0}^{2}}\right)^{1 / 2} .
$$

This approximate expression (36) is valid for values of $\epsilon_{2 r}$ satisfying

$$
Z_{82} / Z_{8 \pm}>1
$$

which is typically about 50 for $0.2 \Omega \mathrm{cm} n$-type germanium.

\section{B. Quarter-wave dielectric disk}

For the high-loss case $\xi_{\text {ig }}$ of Eq. (27) is much greater than unity and consequently (25) becomes

$$
\rho_{g \pm} \simeq 1-2 / \xi_{i g \pm} .
$$

Then

$$
R_{8} \simeq 1 / \xi_{i g}-1 / \xi_{i g+} .
$$

Since

$$
\xi_{i g \pm}=K_{g^{2}}^{2} / K_{g 0} K_{8 \pm},
$$

where $K_{g 2}$ and $K_{g 0}$ are propagation constants in the dielectric region and empty guide respectively, the approximate $R_{g}$ may be derived as

$$
R_{\mathrm{g}} \simeq \frac{\epsilon_{2 r}-\left(K_{c} / K_{0}\right)^{2}}{\left[1-\left(K_{c} / K_{0}\right)^{2}\right]^{1 / 2}} \kappa \mu B\left(\frac{\omega \epsilon_{0}}{\sigma_{s}}\right)^{1 / 2} .
$$

Notice that for the case of no dielectric enhancement 


$$
R_{g}=\left[1-\left(K_{c} / K_{0}\right)^{2}\right]^{1 / 2} \kappa \mu B\left(\omega \epsilon_{0} / \sigma_{s}\right)^{1 / 2} .
$$

$E_{\mathrm{g}}$ for the quarter-wave case is then

$$
E_{g}=\frac{\epsilon_{2 r}-\left(K_{c} / K_{0}\right)^{2}}{1-\left(K_{c} / K_{0}\right)^{2}} .
$$

Comparing Eqs. (40) and (36), the dielectric enhancement by a quarter-wave disk is seen to be increasing more rapidly with $\epsilon_{2 r}$ than by the tapered rod.

The approximations that were made to obtain (39) break down when $\xi_{i g \pm}$ of (37) approaches unity. This occurs when $\epsilon_{2 r}$ approaches a value given by

$$
\epsilon_{2 r}=\left(\sigma_{s} / \omega \epsilon_{0}\right)^{1 / 2}\left[1-\left(K_{c} / K_{0}\right)^{2}\right]^{1 / 2}
$$

Also the magneto-Kerr effect for the guided wave appears to reach its maximum value at a value of $\epsilon_{2 r}$ given by Eq. (41) for the quarter-wave case. The approximation in (41) indicates that when the loss tangent of a semiconductor is large, the relative permittivity of the dielectric should also be large in order to have the maximum enhancement.

The dependence of $R_{g}$ (and $E_{g}$ ) on the thickness of the dielectric slab is shown in Fig. 4. These curves were calculated from Eq. (22) using (25)-(27) for $n$-type germanium with $\mu B=0.1, \sigma_{s} / \omega \epsilon_{s}=15.8, \omega \tau=0.04, \epsilon_{r}=16.27$, and $K_{c}^{2}$ $=33 \mathrm{~cm}^{-2}$.

\section{EXPERIMENTAL RESULTS}

Figure 4 shows the theoretically predicted values of the enhancement factor $E_{g}$ for values of the dielectric relative permittivity ranging from 1 to 9 for the quarter-wave case. Also shown on Fig. 4 are the experimentally measured values of the enhancement factor over the same range of the relative dielectric permittivity. The semiconductor sample used was $0.2 \Omega \mathrm{cm} n$-type germanium. The quarter-wave dielectric slabs used were machined from appropriately mixed stycast material. It was found that attention must be paid to obtaining uniform thickness dielectric samples as

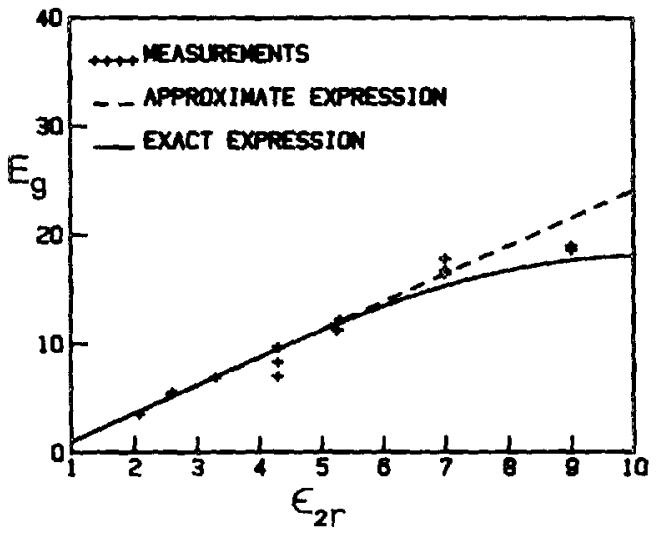

FIC. 4. Enhancement of the magneto-Kerr effect vs disk relative dielectric permittivity for the quarter-wave case.

well as to assuring that the samples were mounted flush to the semiconducting sample. In all cases the sample mount was rotated and the data checked to verify that the dielectric sample was uniform and that the mounting of the sample had been done properly.

As can be seen from Fig. 4, the experimental results match very well with the predicted values. It should be noted that the quality of the sample mount and of the sample homogeneity were especially important at larger values of $\epsilon_{2 r}$.

'M. E. Brodwin and R. J. Vernon, Phys. Rev. 140, 1390 (1965).

${ }^{2}$ B. Lax and S. Zwerdling, Progress in Semiconductors, edited by A. F. Gibson (Academic, New York, 1960), p. 260.

${ }^{3}$ M. E. Brodwin, J. K. Furdyna, and R. J. Vernon, Bull. Am. Phys. Soc. 7, 184 (1962).

${ }^{4}$ R. J. Vernon and T. A. Dorshner, IEEE Trans. Microwave Theory Tech. MTT-19, 287 (1971).

${ }^{5}$ B. Lax and L. M. Roth, Phys. Rev. 98, 548 (1955)

'S. Ramo, J. R. Whinnery, and T. Van Duzer, Fields and Waves in Communication Electronics (Wiley, New York, 1965).

${ }^{7}$ H. Suhl and L. R. Walker, Phys. Rev. 86, 121 (1952).

${ }^{8}$ K. S. Champlin, Physica 28, 1143 (1962). 